\title{
Large-scale gas disk around the radio galaxy Coma $A^{\star}$
}

\author{
R. Morganti ${ }^{1}$, T. A. Oosterloo ${ }^{1}$, S. Tinti ${ }^{2, \star \star}$, C. N. Tadhunter ${ }^{3}$, K. A. Wills ${ }^{3}$, and G. van Moorsel ${ }^{4}$ \\ 1 Netherlands Foundation for Research in Astronomy, PO Box 2, 7990 AA, Dwingeloo, The Netherlands \\ 2 Istituto di Radioastronomia, CNR, via Gobetti 101, 40129 Bologna, Italy \\ 3 Dep. Physics and Astronomy, University of Sheffield, Sheffield, S7 3RH, UK \\ 4 National Radio Astronomy Observatory, Socorro, NM 87801, USA
}

Received 19 December 2002 / Accepted 1 March 2002

\begin{abstract}
We present WSRT and VLA radio observations of the neutral hydrogen in the radio galaxy Coma A. We detect extended H I absorption against both radio lobes of Coma A, at distances of about $30 \mathrm{kpc}$ from the centre. Coma A is the first radio galaxy in which H I is seen in absorption at such large distances from the nucleus. The match between the velocities of the neutral hydrogen and those of the extended ionized gas suggests that they are part of the same disk-like structure of at least $60 \mathrm{kpc}$ in diameter. Most likely, this gas disk is partly ionised by the bulk motion of the radio lobes expanding into it. The gas mass of this disk is at least $10^{9} M_{\odot}$. The relatively regular structure of the gas disk suggests that a merger occurred involving at least one large gas-rich galaxy, at least a few times $10^{8}$ yr ago.
\end{abstract}

Key words. galaxies: ISM - galaxies: active - radio lines: galaxies

\section{Gas in and around radio galaxies}

Many early-type galaxies in the nearby universe appear to have experienced a recent merger/accretion event. In many cases, this has involved at least one gas-rich companion/galaxy that has brought a significant amount of gas into the galaxy. Indeed, H I, FIR and CO observations of elliptical galaxies have demonstrated that many of these objects have an active and interesting cold interstellar medium, often qualitatively (and sometime also quantitatively) similar to that observed in spirals (see Knapp 1999 for a review). Gas is a key factor in determining the evolution of early-type galaxies (e.g. Kauffmann 1996). Among early-type galaxies, gas-rich systems may represent an important phase in the evolution that many elliptical galaxies go through and they give important information on the formation and evolution of these systems.

Apart from being important for the overall evolution of early-type galaxies, there is also compelling morphological and kinematical evidence that the activity in powerful radio galaxies is triggered by galaxy mergers and interactions (e.g. Heckman et al. 1986; Smith \& Heckman 1989; De Koff et al. 1996; Verdoes Klein et al. 1999;

\footnotetext{
Send offprint requests to: $\mathrm{R}$. Morganti,

e-mail: morganti@nfra.nl

* Based on observations with the Westerbork Synthesis Radio Telescope (WSRT) and the Very Large Array (VLA).

** Netherlands Foundation for Research in Astronomy summer student 2001.
}

Capetti et al. 2000; see e.g. Wilson 1996 for a review). This is also supported by the theoretical results (Kauffmann \& Haehnelt 2000) that suggest that the evolution of supermassive black holes is strongly linked to the hierarchical build-up of galaxies. Although these processes are likely to be more efficient and frequent at high redshifts, they are also observed in relatively "nearby" radio galaxies.

If mergers/accretions are indeed important in the evolution/formation of radio galaxies, the question arises what the connection is between their gas properties and those of other early-type galaxies, and whether we can learn something from such a possible connection about the processes that are involved in the AGN activity (Kauffmann \& Haehnelt 2000). Although a general connection merger-AGN activity is reasonably well established, a number of issues is still open, like, for example, 1 ) is the activity triggered by major mergers between gasrich galaxies or by minor accretion events, 2) what is the relationship with other types of merging systems (e.g. the ultra-luminous infra-red galaxies), 3) at what stage of the merger do the jets and the associated activity occur, and 4) are all early-type galaxies likely to go through phases of radio activity?

Powerful radio galaxies are also frequently associated with kpc-sized optical emission line nebulosities, extending up to tens of $\mathrm{kpc}$ from the nucleus. In particular, the ionization of the gas is believed to be due to either anisotropic UV radiation from the active nucleus or to 

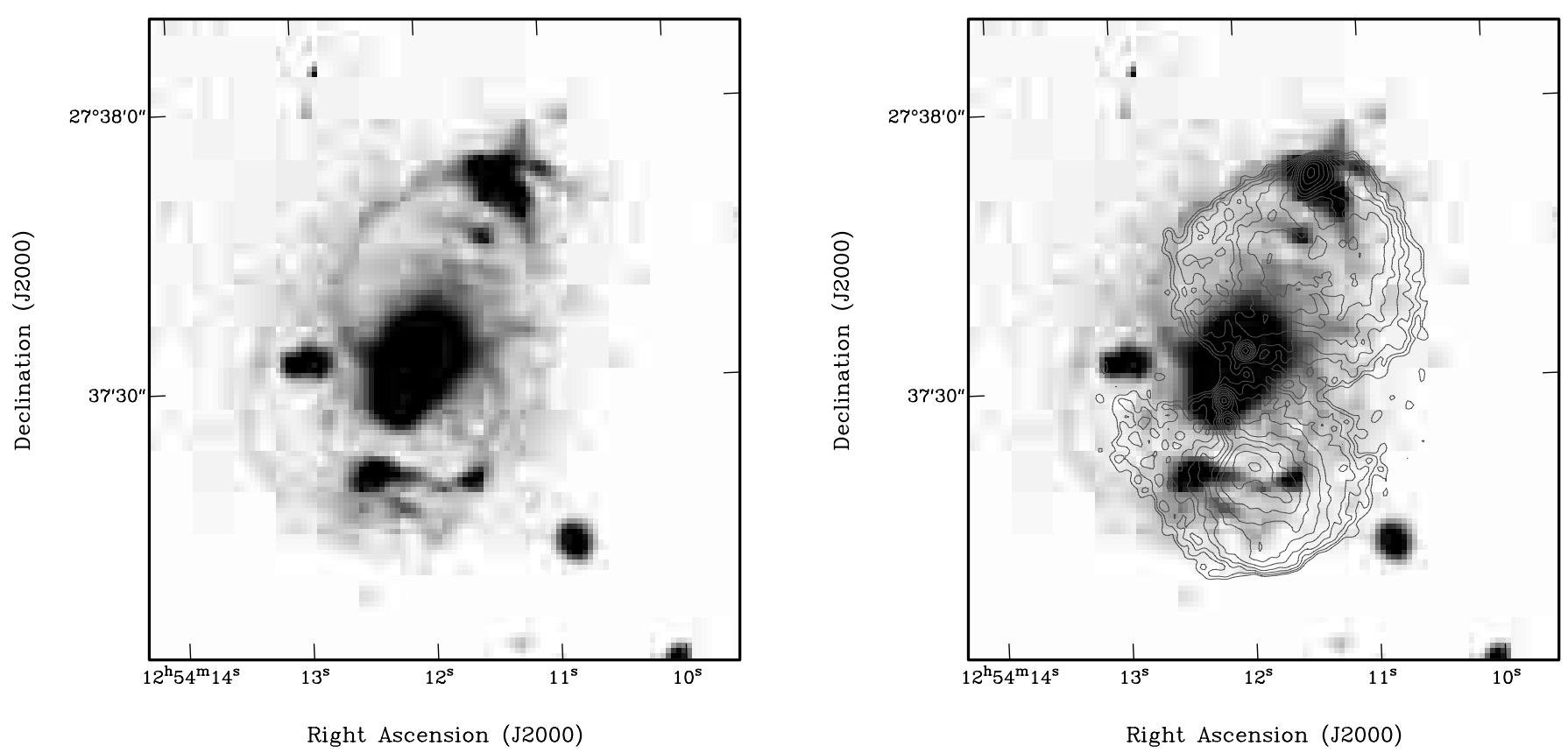

Fig. 1. (Left) H $\alpha$ image of Coma A (from Tadhunter et al. 2000) and (Right) same image with contours of a 20-cm continuum image (about 1 arcsec resolution, from van Breugel et al. 1985) superimposed.

shocks induced by the radio jet and/or the radio lobes. It is likely that in many cases both mechanisms need to be invoked to explain the emission-line properties. One important element that may help to distinguish between the relative importance of the two mechanisms would be to know the distribution of the neutral gas in and around the radio galaxies rather than only the ionization patterns induced by jet or AGN.

It is clear from the above that neutral hydrogen studies can provide a good tool to investigate these different aspects. Usually the H I is too weak to be detected in emission in relatively far away radio galaxies, however certain types of radio galaxies seem to be more promising than others for a possible detection. Among the good candidates is the radio galaxy Coma $\mathrm{A}$. There are several observations that indicate that a complex interaction between the radio structure and a rich interstellar (ISM) medium is occuring in this radio galaxy (Sect. 2). To obtain a more complete knowledge of the ISM in Coma A, we have observed this object in the 21-cm line of HI to try to detect, either in emission or absorption, the gaseous environment of Coma A.

\section{What is known about Coma $A$}

Coma A (3C277.3) is a well-known radio galaxy $(z=$ 0.08579, Clark 1996) studied in detail by e.g. van Breugel et al. (1985). The radio structure and the radio power $\left(\log P=25.82 \mathrm{~W} \mathrm{~Hz}^{-1}\right.$ at $\left.1.4 \mathrm{GHz}\right)$ is characteristic of a radio galaxy intermediate between Fanaroff-Riley type I (FRI) and II (FRII), although Coma A is often classified as FRII. In Fig. 1 the radio contours from $\sim 1$ arcsec resolution VLA observations are shown (see van Breugel et al. 1985 for more details). The radio emission is mainly formed by two "fat" lobes. A bright hotspot is observed only in the northern lobe. The total size is $\sim 100 \mathrm{kpc}^{1}$.

HST observations (Capetti et al. 2000) show a prominent unresolved optical core and patches of dust. A jetcloud interaction is occurring in Coma $\mathrm{A}$ as shown by the cloud of ionised gas observed next to a radio blob just south of the nucleus. This is clearly seen in HST images (Martel et al. 1999; Capetti et al. 2000; Tadhunter et al. in prep.). At this location, the southern radio jet appears to be deflected, it "lights up" and finally it expands into the southern lobe (van Breugel et al. 1985).

A spectacular system of interlocking emission-line arcs and filaments of the same extent as the radio emission has been observed in $\mathrm{H} \alpha$ (Tadhunter et al. 2000). There is a striking match between the radio emission and the morphology of the ionised gas, in particular the optical filament that curves around the northern radio lobe. This suggests that in Coma A the direct interaction with the radio jet ionises the gas, but that also the expansion of the lobe into the environment plays a role, and vice versa that the gaseous environment determines to some extent the morphology of the radio continuum emission.

\section{3. $\mathrm{H}$ I absorption against the radio lobe}

\subsection{WSRT observations}

We have observed Coma A at the frequency of the redshifted H I line (1308.3 MHz) with the WSRT equipped with the DZB backend using a $10 \mathrm{MHz}$ bandwidth, 128 channels, 2 polarisations and all 14 telescopes.

\footnotetext{
1 Throughout this paper we use a Hubble constant of $H_{0}=$ $50 \mathrm{~km} \mathrm{~s}^{-1} \mathrm{Mpc}^{-1}$, and $q_{0}=0$. At the redshift of Coma A 1 arcsec corresponds to $2.2 \mathrm{kpc}$.
} 


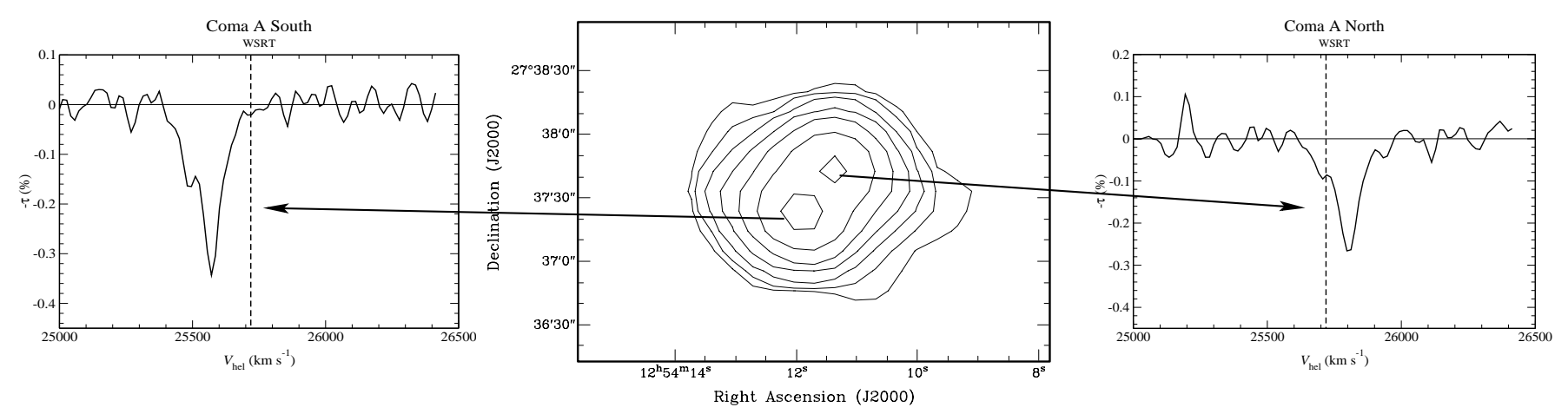

Fig. 2. Profiles of the optical depth $(\tau)$ of the H I absorption against the two radio lobes as detected from the WSRT observations. The contour levels are: $4 \mathrm{mJy}_{\text {beam }}{ }^{-1}$ to $764 \mathrm{mJy}^{\text {beam }}{ }^{-1}$ in steps of factor 2.4 .

The observations were done in 5 observing sessions, four of them of $12 \mathrm{~h}$ (14 Mar. 2000, 20 Mar. 2000, 19 Jul. 2000) while from the last observation (3 Sep. 2000) only $9 \mathrm{~h}$ of good data were obtained due to technical problems. This large amount of time is required in order to reach good sensitivity and investigate the presence of $\mathrm{HI}$ in emission around the radio galaxy.

The data were calibrated and reduced using the Miriad package (Sault et al. 1995) following standard reduction procedures, with the only exception that particular care was taken in checking the stability of the bandpasses of the antennae. This allowed us to reach a spectral dynamic range sufficiently high (better than a few times $10^{4}$ ) to be limited by the thermal noise. The data cube we choose to use was made using a robust-Briggs' weighting (Briggs 1995) with robustness of zero. The noise per channel is $\sim 0.22 \mathrm{mJy} /$ beam and the beam size $38 \times 15$ arcsec with PA $2^{\circ}$. The spectral resolution is $\sim 8.7 \mathrm{~km} \mathrm{~s}^{-1}$. A continuum image was made using the line-free channels. This image was made using uniform weighting (equivalent to a robust image with robustness $=-2$ ) in order to obtain a somewhat higher spatial resolution $(22 \times 11$ arcsec in PA $2^{\circ}$ ).

\subsection{The HI absorption}

The continuum image of Coma $\mathrm{A}$, as made from the WSRT data, is shown in Fig. 2. Given the relatively low spatial resolution, almost all the details visible in Fig. 1 are smoothed out. The two radio lobes are just resolved, while an extended component is also visible. The data clearly show that H I absorption is detected against both lobes at different velocities. This makes Coma A the first radio galaxy known where such absorption is seen at large distances $(\sim 30 \mathrm{kpc})$ from the nucleus. In the southern lobe, the peak absorption is $4.9 \mathrm{mJy}^{\text {beam }}{ }^{-1}$ centred at a velocity of $V_{\text {hel }}=25560 \mathrm{~km} \mathrm{~s}^{-1}$. The width of the absorption is $F W H M \sim 95 \mathrm{~km} \mathrm{~s}^{-1}$. In the northern lobe, the peak absorption is $3.6 \mathrm{mJy}^{\text {beam }}{ }^{-1}$ at a velocity of $V_{\text {hel }}=$ $25780 \mathrm{~km} \mathrm{~s}^{-1}$ and the width is $F W H M \sim 115 \mathrm{~km} \mathrm{~s}^{-1}$. The systemic velocity of Coma $\mathrm{A}$ is $V_{\text {hel }}=25719 \mathrm{~km} \mathrm{~s}^{-1}$
(Clark 1996). Hence, the absorption is blueshifted with respect to the systemic velocity in the southern lobe and redshifted in the northern lobe.

The depth of an absorption line $(\Delta S)$ depends on the optical depth $(\tau)$, the continuum flux density $(S)$ and the covering factor $c_{\mathrm{f}}$ as $\Delta S=c_{\mathrm{f}} S\left(1-e^{\tau}\right)$. Assuming for the moment a covering factor $c_{\mathrm{f}}=1$, the peak optical depths are $\tau=0.35 \%$ and $\tau=0.27 \%$ for the southern and the northern lobe respectively.

The column densities of the neutral hydrogen follow from $N_{\mathrm{HI}}=1.83 \times 10^{18} T_{\text {spin }} \int \tau \mathrm{d} v$ where $T_{\text {spin }}$ is the spin temperature in Kelvin and $v$ is the velocity in $\mathrm{km} \mathrm{s}^{-1}$. Assuming the canonical $T_{\text {spin }}=100 \mathrm{~K}$, we find column densities of $7.2 \times 10^{19}$ atoms $\mathrm{cm}^{-2}$ for the southern lobe and $6.1 \times 10^{19}$ atoms $\mathrm{cm}^{-2}$ for the northern lobe.

\section{3. $\mathrm{HI}$ emission}

No H I was detected in emission in the immediate vicinity of Coma A. The 3- $\sigma$ upper limit to $\mathrm{H} \mathrm{I}$ emission in a single resolution element of the cube is $4.3 \times 10^{19}$ atoms $\mathrm{cm}^{-2}$. Over the width of the absorption lines, the 3- $\sigma$ upper limit is $7-8 \times 10^{19}$ atoms $\mathrm{cm}^{-2}$, more or less the column densities seen in absorption in the WSRT data. Thus, if there was extended $\mathrm{HI}$ emission associated with Coma A it would have been detected (the VLA data show that the actual column densities are somewhat higher, see Sect. 4). It is therefore likely that the extent of the neutral hydrogen associated with Coma $\mathrm{A}$ is limited to the extent of the radio lobes and of the optical emission line gas.

None of the galaxies seen (in projection) near Coma A are detected in emission. At a larger distance $\mathrm{HI}$ is detected at the location of a small galaxy at $\alpha=$ $12^{\mathrm{h}} 54^{\mathrm{m}} 41.8^{\mathrm{s}} \delta=27^{\circ} 30^{\prime} 15.2^{\prime \prime}(\mathrm{J} 2000)$ with $m_{R}=18.8 \pm$ 0.35 (Odewahn et al. 1995). We derive a systemic velocity for this galaxy of $V_{\text {hel }} \sim 25900 \mathrm{~km} \mathrm{~s}^{-1}$ and the H I profile has a $F W H M \sim 300 \mathrm{~km} \mathrm{~s}^{-1}$. This galaxy is situated $\sim 15^{\prime}$ from Coma A, corresponding to almost $2 \mathrm{Mpc}$. Thus, no interaction is expected between this galaxy and Coma A. The H I mass is substantial: $M_{\mathrm{HI}} \sim 10^{10} M_{\odot}$. 


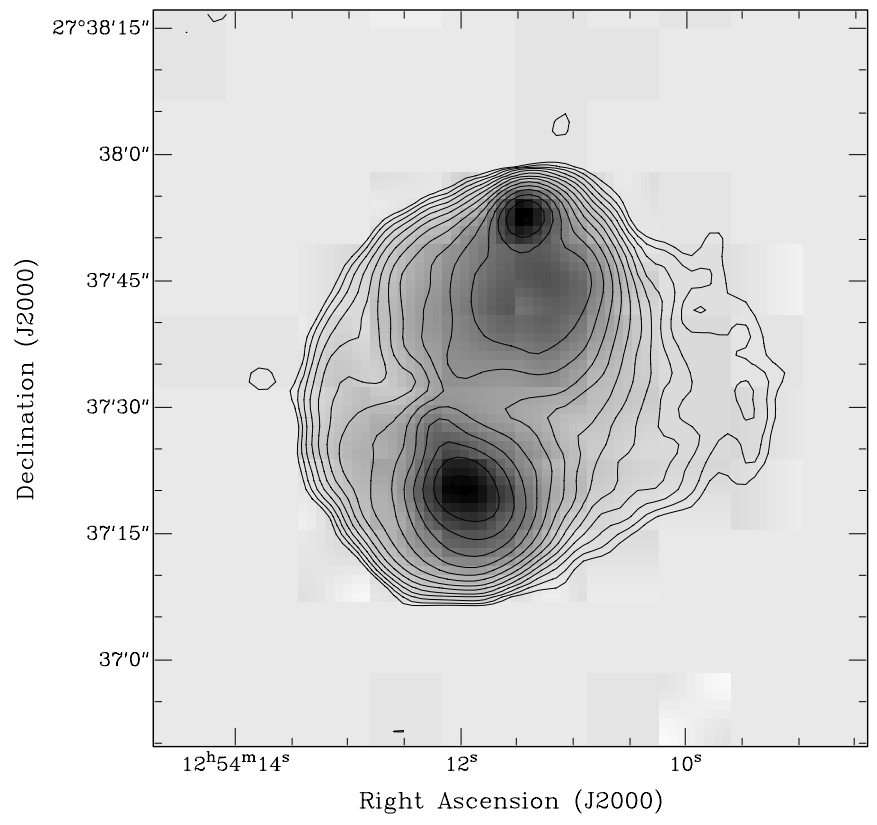

Fig. 3. Contours of the radio continuum at the resolution of $4.5 \times 4 \operatorname{arcsec}\left(\mathrm{PA}-70^{\circ}\right)$. The contours for the continuum range from $1 \mathrm{mJy}^{\mathrm{beam}}{ }^{-1}$ to $1 \mathrm{Jy}$ beam $^{-1}$ in steps of factor 1.5 .

\section{The spatial distribution of the $\mathrm{HI}$ absorption}

\subsection{VLA observations}

To be better able to locate where the H I is occurring, we have observed Coma A also with the VLA in its $B$ configuration. These observations were carried out on 15/16 May 2001. In order to have sufficient velocity resolution and to cover a large enough velocity range, we used a bandwidth of $3.125 \mathrm{MHz}$ and 64 channels for each of the two bands available. The two bands were placed with some overlap so that a total band of about $6 \mathrm{MHz}$ is covered. A velocity resolution of $12 \mathrm{~km} \mathrm{~s}^{-1}$ is obtained using this setup. The observation resulted in 6 hours of good quality data while the data of four hours of observations could not be used due to technical problems.

Also the VLA data were reduced using the Miriad package, again using standard reduction procedures. Initially, two data cubes (one for each band) were made using a robust-Briggs' weighting of zero (Briggs 1995). This gives a spatial resolution of $4.5 \times 4.0 \operatorname{arcsec}\left(\mathrm{PA}-70^{\circ}\right)$. The noise in a single channel is $0.62 \mathrm{mJy}^{\text {beam }}{ }^{-1}$. At this resolution, H I absorption is only weakly detected, especially in the northern lobe. We therefore have produced data cubes by tapering the visibilities in order to improve the sensitivity for more diffuse absorption. For the southern lobe, we have tapered the data with a Gaussian of $F W H M=8$ arcsec, resulting in a beam of $9.4 \times 9.0$ $\left(\mathrm{PA} 69^{\circ}\right.$ ) and a rms noise of $0.78 \mathrm{mJy}$ beam $^{-1}$ in a single channel. A Gaussian taper of $F W H M=10$ arcsec was necessary to image the region of the $\mathrm{HI}$ absorption in the northern lobe. The resulting beam is $11.5 \times 11.0\left(\mathrm{PA} 66^{\circ}\right)$ and the rms noise is $0.85 \mathrm{mJy}^{\text {beam }}{ }^{-1}$ per channel.

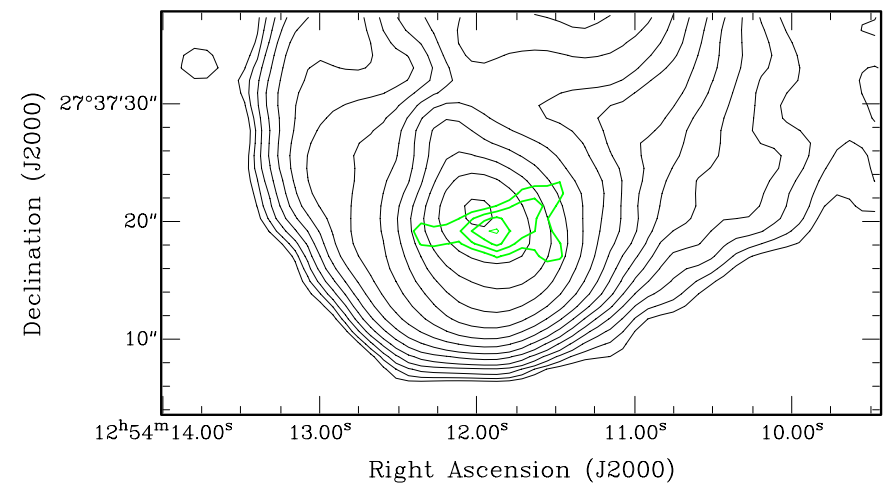

Fig. 4. Contours of the radio continuum (black) and contours of column density derived from the H I absorption (grey contours) obtained from the highest resolution data cube in the southern lobe (see text). The contours for the continuum range from $1 \mathrm{mJy}^{\text {beam }}{ }^{-1}$ to $1 \mathrm{Jy}_{\text {beam }}{ }^{-1}$ in steps of a factor 1.5. The contours of the $\mathrm{H}$ I absorption range from 0.4 to $1.0 \times 10^{20}$ atoms $\mathrm{cm}^{-2}$ in step of $0.2 \times 10^{20}$ atoms $\mathrm{cm}^{-2}$.

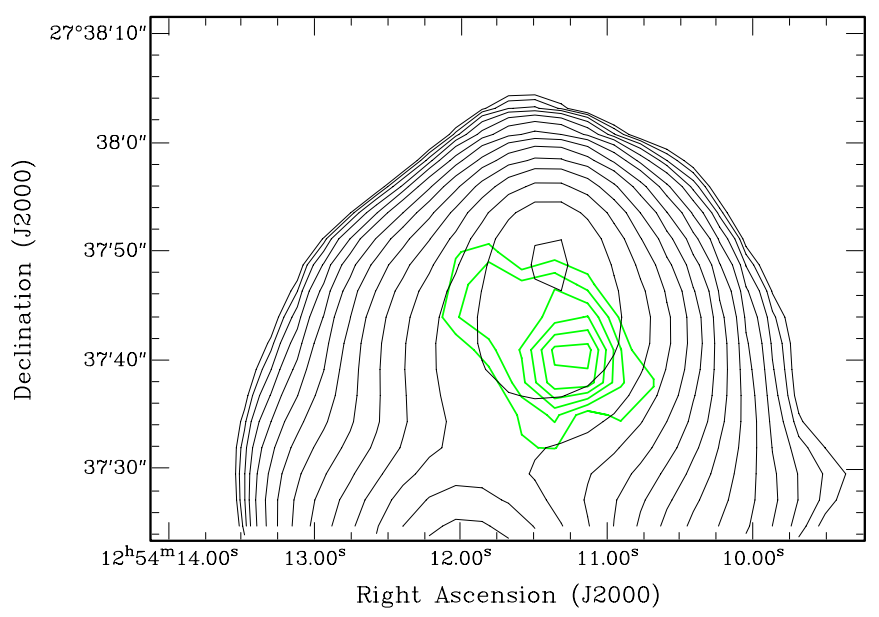

Fig. 5. Contours of the radio continuum (black) and contours of column density derived from the H I absorption (grey contours) obtained from the highest resolution data cube in the southern lobe (see text). The contours for the continuum range from $2 \mathrm{mJy}^{\text {beam }}{ }^{-1}$ to $1 \mathrm{Jy}_{\text {beam }}{ }^{-1}$ in step of factor 1.5. The contours of the $\mathrm{HI}$ absorption range from 0.4 to $0.9 \times 10^{20}$ atoms $\mathrm{cm}^{-2}$ in step of $0.1 \times 10^{20}$ atoms $\mathrm{cm}^{-2}$.

A continuum image was also obtained using the linefree channels taken from one of the observing bands. The noise of the continuum image made with robust-Briggs' weighting of zero is $0.37 \mathrm{mJy}^{\text {beam }^{-1}}$.

\subsection{The location of the $\mathrm{HI}$ absorption}

In Fig. 3 the continuum image as made from the VLA data is shown. Much more detail is visible compared to the image made from the WSRT data. The two radio lobes are now well resolved, while still much of the extended component that is visible in the WSRT image, but not in the image made with the VLA A array, is detected.

In the full resolution VLA data cubes very little absorption is reliably detected. No absorption is detected 


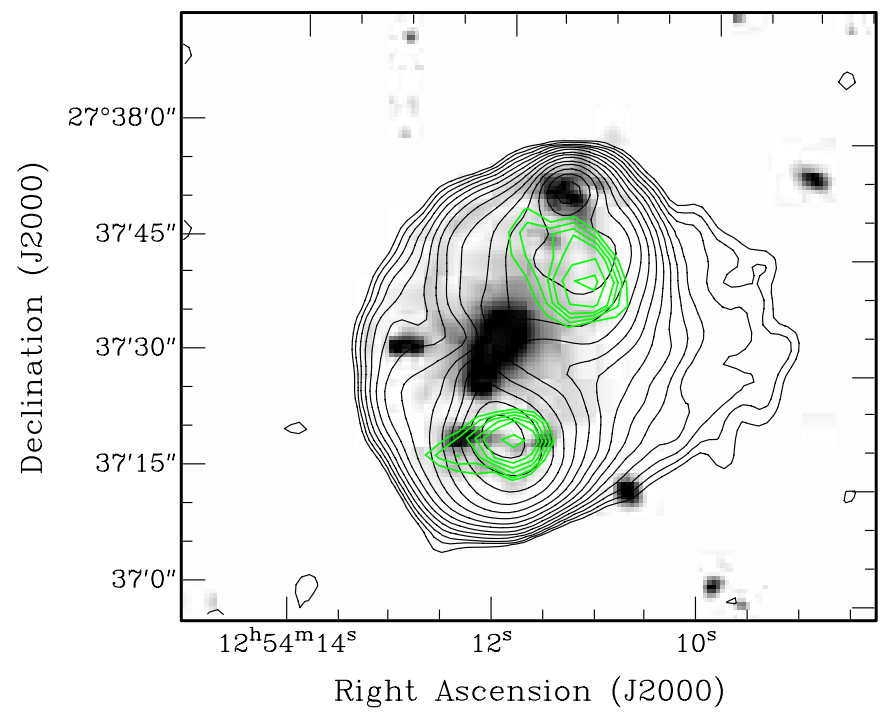

Fig. 6. $\mathrm{H} \alpha$ image (grey scale) with superimposed the contours of the radio continuum (black) and the contours of column density derived from the $\mathrm{H}$ I absorption (grey). In the northern lobe the contours range from 0.35 to $0.85 \times 10^{20}$ atoms $\mathrm{cm}^{-2}$ in step of $0.1 \times 10^{20}$ atoms $\mathrm{cm}^{-2}$. In the southern lobe the contours range from 1 to $1.5 \times 10^{20}$ atoms $\mathrm{cm}^{-2}$ in step of $0.1 \times 10^{20}$ atoms $\mathrm{cm}^{-2}$.

against the nucleus nor against the location just south of the nucleus where the jet-cloud interaction is occurring (van Bruegel et al. 1985), with upper limits to the optical depth of 4 and $2.7 \%$ respectively.

In the southern lobe the peak absorption is detected only at the $3-\sigma$ level at $2.9 \mathrm{mJy}^{\text {beam }}{ }^{-1}$ in a single channel. This corresponds to a maximum optical depth of $\tau=1.7 \%$. In Fig. 4 the integrated H I absorption is shown (grey contours) at the full resolution. The absorption is found near the region with the brightest continuum emission, with a suggestion that the absorption occurring just $\mathrm{SW}$ of this maximum in the continuum. No absorption is detected above the 3- $\sigma$ level in the northern lobe. These results indicate that the $\mathrm{HI}$ absorption is due to an $e x-$ tended screen in front of the radio lobes.

In order to improve the sensitivity for extended absorption lower resolution data cubes were made as described in Sect. 4.1. In these lower-resolution cubes absorption is detected against both radio lobes. Figure 5 shows where this absorption is detected in the northern radio lobe (at the resolution of the tapered data). The grey contours represent the integrated $\mathrm{HI}$ absorption. The absorption is somewhat extended and is not occurring near the continuum hotspot. It is clearly displaced from it, in the direction of the nucleus.

In Fig. 6 the contours of the H I absorption detected against both radio lobes (at the resolution of the tapered data) are shown, superimposed on the $\mathrm{H} \alpha$ image.

The peak absorption in the southern lobe is $6.2 \mathrm{mJy} \mathrm{beam}^{-1}$ which corresponds to an optical depth of $\tau_{\max }=1 \%$. This is higher than in the WSRT data, but this can be explained by the difference in

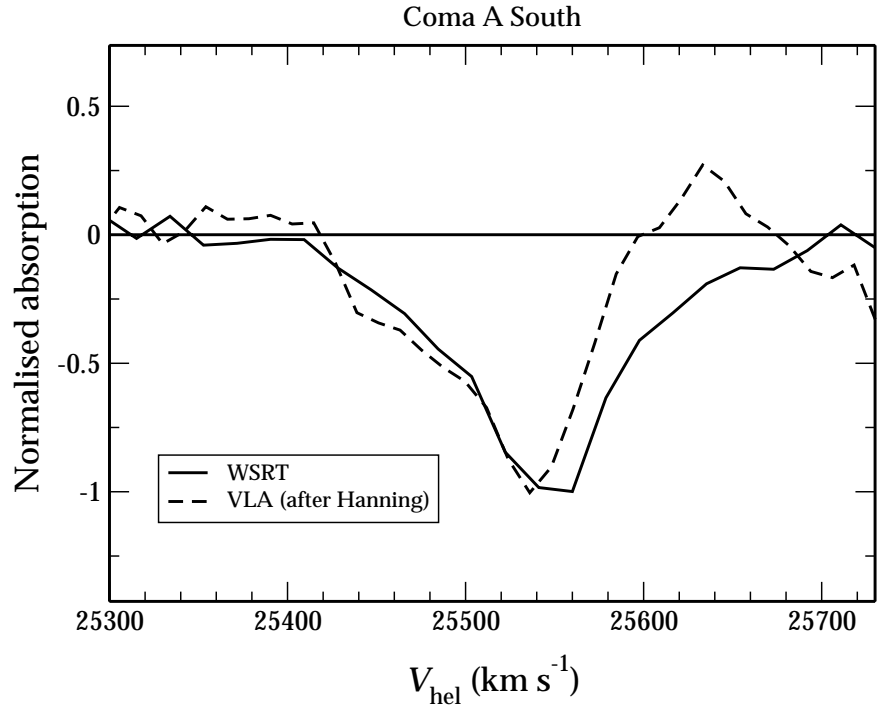

Fig. 7. Comparison between the profile of the south lobe obtained with WSRT (long dash) and the VLA after tapering (short dash).

velocity resolution. The width of the absorption profile is $F W H M \sim 70 \mathrm{~km} \mathrm{~s}^{-1}$. For the northern lobe the peak of the absorption is $4.2 \mathrm{mJy}^{\text {beam }^{-}} 1\left(\tau_{\max }=0.9 \%\right)$ and $F W H M \sim 60 \mathrm{~km} \mathrm{~s}^{-1}$. The corresponding column densities are $1.3 \times 10^{20}$ atoms $\mathrm{cm}^{-2}$ for the southern lobe and $8.7 \times 10^{19}$ atoms $\mathrm{cm}^{-2}$ for the northern lobe, assuming $T_{\text {spin }}=100 \mathrm{~K}$. By integrating over the area where the absorption is occurring, the estimated HI mass is found to be about $M_{\mathrm{H} \text { I }} \simeq 10^{9} M_{\odot}$.

When comparing with the WSRT results one sees that the values of the peak absorption are, within the errors, the same, but that the widths of the absorption profiles are smaller in the VLA data (see Fig. 7). The VLA observations recover about $70-75 \%$ of the absorption detected in the WSRT data. This, and the larger widths of the WSRT profiles implies that more absorption, at a level too faint to be detected in the VLA observations, is present over a larger region of the lobes, at somewhat different velocities. The absorption therefore covers a significant fraction of both radio lobes.

\section{Neutral and ionised gas: A possible large-scale gas disk}

The data discussed above show that there is H I absorption in front of both radio lobes of Coma A. This makes Coma A quite unique: the absorption is not detected against the nuclear regions of a radio galaxy but it is situated at large distances (tens of $\mathrm{kpc}$ ) from the centre.

The absorption covers a significant fraction of both radio lobes. The strongest absorption is localised in areas at about $15 \operatorname{arcsec}(\sim 33 \mathrm{kpc})$ from the radio core. We do not detect $\mathrm{HI}$ absorption against some of the brighter regions of radio emission, e.g. the hot spot in the northern lobe and the bright knot just south of the nucleus. 


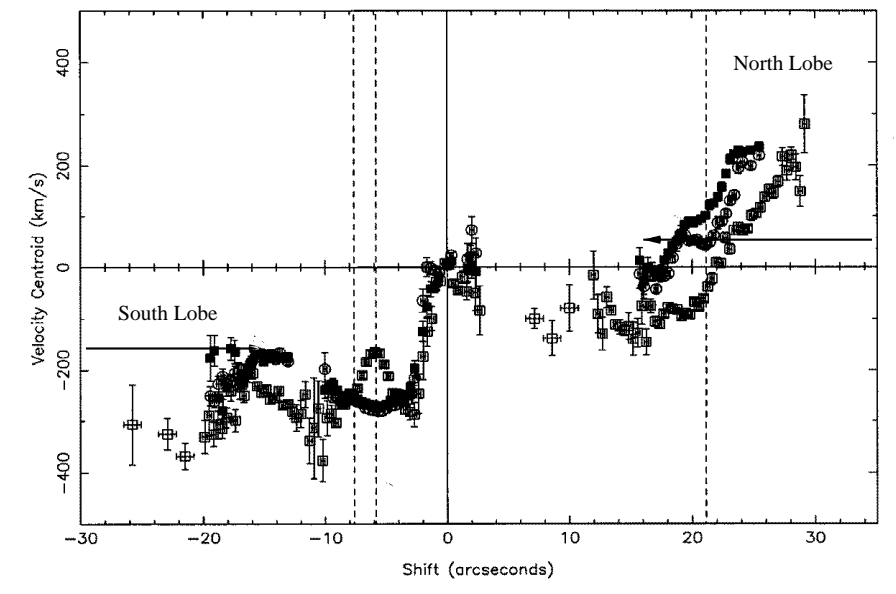

Fig. 8. Plot of the optical velocities along the radio axis derived from $\mathrm{H} \alpha$ (filled squares) and [O III] (open squares) emission lines. The velocities derived from the H I absorption against the two radio lobes are marked.

It is important to understand the relationship between the neutral hydrogen detected at large distances from the centre and the large structure of ionised gas found by Tadhunter et al. (2000). For this it is interesting to compare the velocities of the HI with those of the ionised gas (from Clark 1996). These optical data consist of longslit spectra taken along the major axis of the ionised-gas distribution. These data do not cover exactly the same regions where the $\mathrm{HI}$ absorption is detected, but the difference in location is not too large and a comparison is still meaningful.

The match between the optical velocities and those of the $\mathrm{HI}$ is very good (Fig. 8). Both the ionised gas and the neutral gas are blueshifted, by about the same amount, south of the centre and redshifted north of the centre. This close correspondence in velocity between the ionised and neutral gas demonstrates that they are two phases of the same gas structure.

From the morphology of the ionised gas, Tadhunter et al. (2000) argued that Coma A could have experienced a recent merger and the filamentary structure of the ionised gas would represent pre-existing gas structures. The large amount of neutral gas detected in this galaxy via $\mathrm{H} \mathrm{I}$ absorption, supports the idea that Coma A is likely to have experienced a merger. Considering that a large fraction of the total gas mass must be ionised, this merger must have involved at least several times $10^{9} M_{\odot}$ of gas. The merger in Coma A must have involved at least a reasonably sized gas-rich galaxy.

A number of faint galaxies are found within $100 \mathrm{kpc}$ of the nucleus of Coma A and could be associated with the extended $\mathrm{H} \alpha$ filamentary structures. One of them (see Fig. 1 in Tadhunter et al. 2000) is situated (in projection) on the western edge of the southern H I cloud. This galaxy, even if spectroscopically confirmed to be a real companion, appears too small to be the donor of the observed gas because of the large amount of gas detected around Coma A and the large extent over which this gas is found.
One of the aims of the observations presented here was to look for very large-scale H I tails around Coma A. These structures are often observed in recent mergers (e.g. Yun et al. 1994; Hibbard \& van Gorkom 1996; Hibbard \& Mihos 1995). No such structures have been detected in Coma A. The gas acquired is apparently reasonably settled in an area of a few tens of kpc, instead of the hundred kpc long structures often seen in on-going mergers. Moreover, the kinematics and the more or less symmetric distribution of the gas, suggest that the gas has settled at least to some extent. Considering that this settling, given the size and the velocities observed, would take roughly a few times $10^{8} \mathrm{yrs}$, the merger in Coma $\mathrm{A}$ is likely to have happened several times $10^{8}$ yrs ago.

An important constraint to consider in the interpretation is that $\mathrm{HI}$ absorption is detected against both radio lobes. Neutral gas must be, therefore, present in front of both of them. Thus, to explain the observed characteristics of both the ionised gas and the neutral hydrogen absorption in Coma $\mathrm{A}$, we have to assume that if the gas is distributed in a disk-like structure, the radio plasma is actually expanding in this disk. The distribution of the ionised gas supports this. The morphology of the ionised gas appears to be distorted by the interaction with the radio lobes, e.g. with shocks fronts sweeping up material into the filamentary structures observed. The fact that the neutral hydrogen seems to show quite broad absorption profiles could be an indication of a kinematical disturbance as the effect of interaction between the radio and the ISM. A first-order anti-correlation is observed between the ionised and the neutral gas, in particular in the northern lobe.

It is also interesting to note that we find the $\mathrm{HI}$ in the region where we would expect the gas to be ionised if there is an illuminating quasar. Therefore, the H I measurements provide further evidence for the idea that the quasar illumination in Coma A is weak or absent (Tadhunter et al. 2000).

In many cases where a gas/dust disk is observed in a radio galaxy, the radio axis is perpendicular to this disk. However, this is not a golden rule and applies mainly to small dust lanes near the nucleus (e.g. de Koff et al. 2000; de Ruiter et al. 2001). Moreover, several examples exist, perhaps related to the tri-axial nature of the mass distribution, where the inner gas disk is perpendicular to the outer gas disk (e.g. NGC 5266, Morganti et al. 1997; NGC 2685, Oosterloo et al. (in prep.) and possibly also Centaurus A, Schiminovich et al. 1994). In some of these galaxies the radio jet is directed into the plane of the outer gas disk and interacting with this gas disk. A spectacular example of this is the Seyfert galaxy IC 5063 (Morganti et al. 1998). Hence, a geometry with the lobes expanding into the outer gas disk can be expected and is actually observed in some cases.

Note that in the radio continuum, apart from the two lobes, there are extensions to the east and the west with the western extension larger than the eastern one. The morphology of the ionised gas suggests that the interaction on the eastern side is stronger than on the western side. 
The larger western extension of the radio continuum on suggests that the gas disk plays a role in confining and shaping the radio continuum structure.

\section{How unique is Coma $A$ ?}

Large disks of neutral gas have already been observed in a number of early-type galaxies (see e.g. Oosterloo et al. 2001a,b and ref. therein; van Gorkom 1997 and ref. therein) and the detection of such a disk in Coma A fits naturally in the scenario that these large disks and the AGN activity are merger related. The main difference of Coma A with the galaxies mentioned above is the higher radio continuum power. Such extended gas disks are very difficult to detect if the galaxy is at a relatively large redshift (as most of the radio galaxies are), but our work on Coma A shows that such disks can be studied through $\mathrm{H}$ I absorption, an observational technique that can, with current radio telescopes, be used out to redshift $\sim 3.5$.

The only other objects known to us that appears to show characteristics of the neutral gas distribution similar to Coma A are 3C 433 and 3C 234. Recent VLA observations (Morganti et al. 2001) show that at least part of the H I absorption (detected originally with the Arecibo telescope, Mirabel 1989) is situated against the southern radio lobe at about $40 \mathrm{kpc}$ from the nucleus. Unlike Coma A, no ionised gas has been detected in 3C 433 at such a distance from the centre. In $3 \mathrm{C} 234 \mathrm{H} \mathrm{I}$ absorption has been detected toward the NE lobe at about 50 arcsec from the centre (Pihlström 2001).

On a smaller scale, situations similar to Coma A can be found in a handful of objects. We want to stress that in these cases the scale of the phenomenon is at most a few kpc while in Coma A the absorption is observed at tens of kpc from the nucleus.

One of these objects is PKS 2322-123, the central radio galaxy in the cooling flow cluster Abell 2597 studied by O'Dea et al. (1994) and Taylor et al. (1999). Spatially extended 21-cm H I absorption is detected against this source (O'Dea et al. 1994) indicating that the radio lobes are surrounded by a collection of clouds containing both neutral and ionised gas. Interestingly this object also shows evidence of optical arcs associated with the radio lobes, as observed in the case of Coma A. Koekemoer et al. (1999) suggest that the properties of this source are explained in terms of accretion of gas by the $\mathrm{cD}$ during the interaction with a gas-rich galaxy. The same could apply to Coma A, with the main difference that in PKS 2322-123 it occurs at only a few kpc from the nucleus.

Two more examples where the HI absorption is observed in coincidence with the radio lobes instead of the radio core are IC 5063 (Morganti et al. 1998; Oosterloo et al. 2000) and 3C 236 (Conway \& Schilizzi 2000). Again, in both cases the distance from the nucleus of the H I absorption is only a few kpc. These are examples of a jetcloud interaction between the radio plasma and the ISM. It is interesting to note that in IC 5063 (and likely also in
$3 \mathrm{C} 236)$ the radio axis is parallel to the axis of the largescale gas disk, as we claim could be the case in Coma A.

More radio galaxies with very extended neutral gas distributions are likely to exist. Extended H I absorption (observed against the $\operatorname{Ly} \alpha$ emission) has been found in a high fraction of high- $z$ radio galaxies (van Ojik et al. 1997). This is considered an indication that high- $z$ radio galaxies are located in dense environments and is a diagnostic for the effects of radio jet propagation in this dense medium. Although this phenomenon may be occurring more frequently and more efficiently at high redshifts, in the low redshift radio galaxies described here we may witnessing a similar situation. As the sensitivity of the radio telescopes improves, such extended gas distributions will be found in more radio galaxies. We have recently detected a large disk-like H I structure in the compact radio galaxy B2 0648+27 (Morganti et al. 2001). In this object the radio source is unresolved with arcsecond resolution and therefore $\mathrm{HI}$ absorption is only detected against the central source. However, H I in emission is detected out to a radius of $\sim 80 \mathrm{kpc}$.

\section{Summary}

We have detected $\mathrm{H}$ I absorption against the radio lobes of the radio galaxy Coma $\mathrm{A}$. This absorption covers a significant fraction of these lobes and occurs at large distances from the nucleus $(\sim 33 \mathrm{kpc})$. The kinematics of the H I matches that of the large structure of ionised gas present in Coma $\mathrm{A}$ and the ionised and the neutral gas are two phases of the same structure. No H I is detected in emission outside the area covered by the lobes. The fact that we detect absorption on both sides from the nucleus suggests that the radio lobes are expanding into a large gas disk. The morphology of the ionised gas strongly supports this.

The gas disk appears relatively settled indicating that the AGN activity, assuming the period of such activity lasts at most a few times $10^{7} \mathrm{yrs}$, started at least $10^{8} \mathrm{yrs}$ after the merger. The mass of the gas involved in this merger is at least $10^{9} M_{\odot}$, suggesting that at least one reasonably-sized gas-rich galaxy was involved.

Acknowledgements. The WSRT is operated by the ASTRON (Netherlands Foundation for Research in Astronomy) with support from the Netherlands Foundation for Scientific Research (NWO). The VLA is a facility of the National Radio Astronomy Observatory, which is operated by Associated Universities Inc., under cooperative agreement with the National Science Foundation.

\section{References}

Briggs, D. 1995, Ph.D. Thesis, New Mexico Institute of Mining and Technology

Capetti, A., de Ruiter, H. R., Fanti, R., et al. 2000, A\&A, 362, 871

Clark, N. E. 1996, Ph.D. Thesis, University of Sheffield

Conway, J. E., \& Schilizzi, R. T. 2000, in Proc. of the 5th European VLBI Network Symp., ed. J. E. Conway, A. G. Polatidis, R. S. Booth, \& Y. M. Pihlström, published Onsala Space Observatory, ISBN 91-631-0548-9, 123 
de Koff, S., Baum, S. A., Sparks, W. B., et al. 1996, ApJS, 107, 621

de Koff, S., Best, P., Baum, S., et al. 2000, ApJS, 129, 33

Heckman, T. M., Balick, B., van Breugel, W. J. M., \& Miley, G. K. 1983, AJ, 88, 583

Hibbard, J. E., \& Mihos, J. C. 1995, AJ, 110, 140

Hibbard, J. E., \& van Gorkom, J. H. 1996, AJ, 111, 655

Kauffmann, G. 1996, MNRAS, 281, 487

Kauffmann, G., \& Haehnelt, M. 2000, MNRAS, 311, 576

Knapp, G. R. 1999, in Star Formation in Early-Type Galaxies, ed. P. Carral, \& J. Cepa, ASP Conf. Proc., 119

Koekemoer, A. M., O'Dea, C. P., Sarazin, C. L., et al. 1999, ApJ, 525, 621

Martel, A. R., Baum, S. A., Spark, W. B., et al. 1999, ApJS, 122,81

Mirabel, I. F. 1989, ApJ, 340, L13

Morganti, R., Oosterloo, T. A., \& Tzvetanov, Z. 1998, AJ, 115, 915

Morganti, R., Sadler, E. M., Oosterloo, T., Bertola, F., \& Pizzella, A. 1997, AJ, 113, 937

Morganti, R., Oosterloo, T. A., Tinti, et al. 2001, in Seeing through the dust, ed. R. Taylor, T. Landecker, \& A. Willis, ASP, in press [astro-ph/0112269]

Odewahn, S. C., \& Aldering, G. 1995, AJ, 110, 2009

Oosterloo, T. A., Morganti, R., Tzioumis, A., et al. 2000, AJ, 119,2085

Oosterloo, T., Morganti, R., Vergani, D., Sadler, E. M., \& Caldwell, N. 2001a, AJ, in press [astro-ph/0111141]

Oosterloo, T., Morganti, R., \& Sadler, E. M. 2001b, in Gas and Galaxy Evolution, ed. J. E. Hibbard, M. Rupen, \& J. H. van Gorkom, ASP Conf. Proc., 240, 251 [astro-ph/0009112]
O'Dea, C., Baum, S., \& Gallimore, J. 1994, ApJ, 436, 669

Pihlström, Y. M. 2001, Ph.D. Thesis, Chalmers University \& Onsala Observatory

de Ruiter, Parma, P., Fanti, R., Capetti, A., \& Morganti, R. 2001, in The central kpc of starburst and AGN, ed. J. H. Knaper, J. E. Beckman, I. Shlosman, \& T. J. Mahoney, ASP Conf. Ser, in press [astro-ph/0106490]

Sault, R. J., Teuben, P. J., \& Wright, M. C. H. 1995, in Astronomical Data Analysis Software and Systems IV, ed. R. Shaw, H. E. Payne, \& J. J. E. Hayes, Astronomical Society of the Pacific Conf. Ser., 77, 433

Schiminovich, D., van Gorkom, J. H., van der Hulst, J. M., \& Kasow, S. 1994, ApJ, 423, L101

Smith, E. P., \& Heckman, T. M. 1989, ApJ, 341, 658

Tadhunter, C. N., Villar-Martin, M., Morganti, R., \& Axon, D. 2000, MNRAS, 314, 849

Taylor, G. B., O'Dea, C. P., Peck, A. B., \& Koekemoer, A. M. 1999, ApJ, 512, L27

van Breugel, W., Miley, G., Heckman, T., Butcher, H., \& Bridle, A. 1985, ApJ, 290, 496

van Gorkom, J. 1997, in The Nature of Elliptical Galaxies, ed. M. Arnaboldi, G. S. Da Costa, \& P. Saha, ASP Conf. Ser., 116,310

Verdoes Klein, G. A., Baum, S. A., de Zeeuw, P. T., \& O'Dea, C. P. 1999, AJ, 118, 2592

van Ojik, R., Röttgering, H., Miley, G., \& Hunstead, R. 1997, A\&A, 317, 358

Wilson, A. 1996, in Energy Transport in radio galaxies and quasars, ed. P. E. Hardee, A. H. Bridle, \& A. J. Zensus, ASP Conf. Ser., 9

Yun, M. S., Ho, P. T. P., \& Lo, K. Y. 1994, Nature, 272, 530 